\title{
Proximity-Based Adaptation of Content to Groups of Viewers of Public Displays
}

\author{
Amir E. Sarabadani Tafreshi, Kim Marbach, and Gerhard Tröster \\ ETH Zürich University, CH-8092 Zürich, Switzerland
}

\begin{abstract}
Responsive design adapts web content to different viewing contexts to deliver an optimal viewing and interaction experience. Recent work proposed a model and framework for proximity-based adaptation of web content as a new dimension for responsive web design. While it was shown that the model improves the perception and user engagement for single viewers, until now, the effect had not been investigated for multiple simultaneous viewers who may be at different distances from the display. In this paper, we report on an initial study that evaluated and compared the effects of using the average distance of viewers as the basis for handling adaptation of content to multiple viewers with a classic one that adapts content based only on display characteristics. Our results show that the adaptive model provides a better view of the content and improves user engagement, but can be confusing when serving multiple viewers.
\end{abstract}

\section{KEYWORDS}

Distance; large displays; Responsive design; multiple viewers.

\section{Introduction}

Despite the fact that viewers of public displays perceive the content of a display at different sizes according to their distance from the display, current responsive web designs (RWD) only adapt web content based on the characteristics of the device and browser. Therefore, in a recent work, Tafreshi et al. [1] proposed a model that extends current responsive design techniques to take user proximity into account as part of the viewing context for public displays. However, one of the open issues and major challenges is considering how to handle the fact that public displays typically have multiple simultaneous viewers who may be at different distances from the display.

The proposed JavaScript framework, ResponDis, was designed to support experimentation with variants of proximity-based adaptation model in both single and multi-viewer contexts. However, until now only single-user scenarios had been investigated. In this paper, we report on our initial investigations of multi-viewer contexts. Following one of the suggested methods by Tafreshi et al. [1], we use the average viewer distance of viewers to calculate the proximity of a group of users to a display. Our hypothesis is that the average-based method that considers the proximity of all viewers would improve the viewers' perception and user engagement in general. A user study with 24 participants was carried out to compare the resulting dynamic proximity-based adaptation of screen content with an approach that performs a static adaptation of content based solely on the 
display characteristics. Our findings show that the proximity-based adaptive model improved the user engagement and provided a better view of the content that avoided users having to move close to the display. However, we observed that the adaptive approach resulted in some confusion.

\section{Related Work}

HTML5 and CSS3 provide features that facilitate responsive design in which a website adapts to the characteristics of a device or browser. Recent proposals for CSS4 media queries take support for responsive design further by catering to other characteristics of the viewing context, for example adjusting the design depending on the luminosity level [2]. Recent works $[1,3]$ add another dimensions by also taking into account the distance and walking-speed of the viewer as important factors for responsive web design, especially in the case of public displays.

In all of these cases, each of the factors taken into account is associated with a single input. For example, the size of the browser, the level of ambient light, or the distance of the viewer to the display. However, since public displays can often have multiple viewers, there could be multiple inputs for viewer distance. Proximity-based responsive web designs therefore need to somehow mediate in the case of multiple viewers. Strategies previously proposed or envisaged in the literature to handle such situations can be classified into three approaches: (i) split-screen, (ii) selecting one target viewer out of a group, and (iii) mixed.

A split-screen approach $[4,5]$ splits the screen into multiple regions and allocates each of them to a different viewer. Clearly, increasing the number of viewers leads to these regions having smaller sizes which can negatively affect the perception of the viewers to the content and also distract them. At the same time, it can waste screen space if regions end up showing the same information.

The second approach has to decide on a strategy for selecting one of the viewers as a target, thereby ignoring the others and adapting the content based on the distance of the target viewer to the display [6]. One possibility would be to take the user first detected as the target, while another is to select the viewer closest to the display.

The mixed approach [6] combines the distance of all the viewers and represents them as a single parameter for design decisions. For example, the average distance of the viewers could be used. However, it is not clear whether such an approach improves the viewers' experience, so we chose to investigate this.

\section{Extension of the Model for Multiple Viewers}

The proximity-based adaptation model [1] provides the optimal content resolution (CVR) for a viewer who is in front of a display. The calculation of optimal content resolution is not only based on the viewer's distance to the display (VD) and the display's diagonal size (DS) in inches but also on the display's native horizontal and vertical resolution in pixels, denoted NHR and NVR, respectively. The equation that considers all of these factors to calculate the CVR is equal to: 


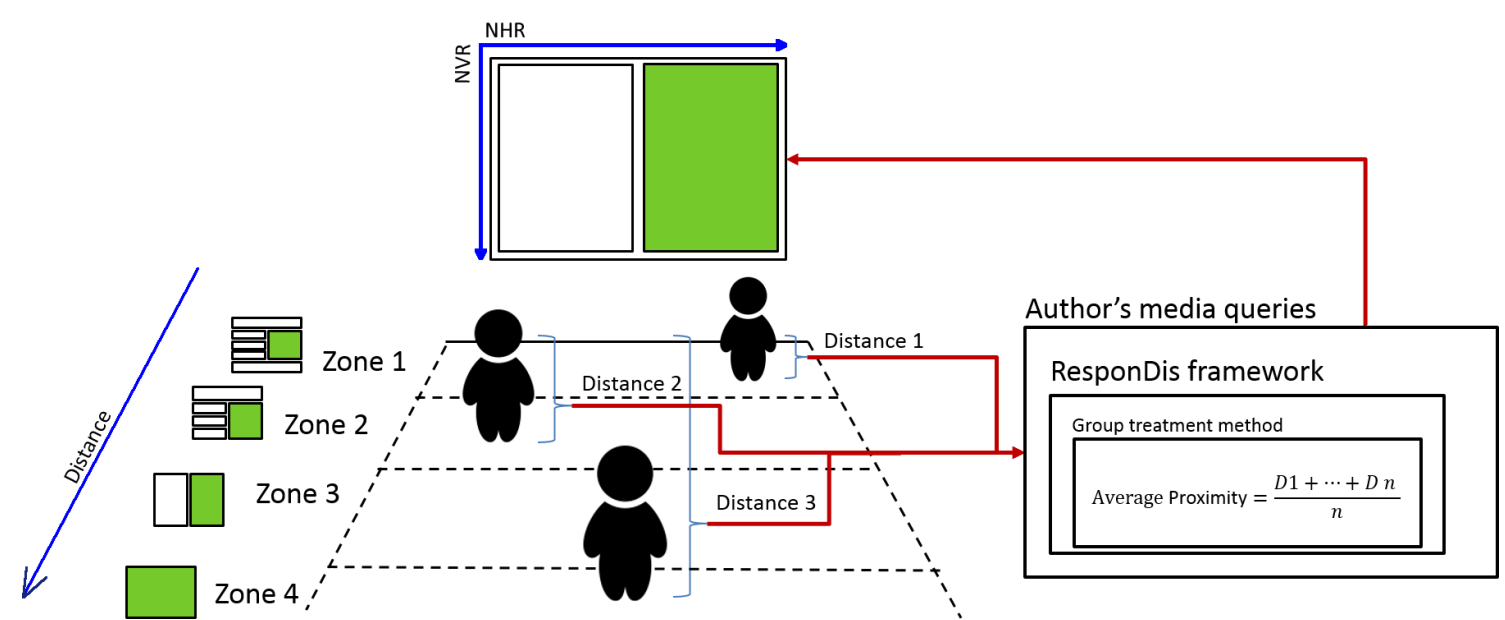

Figure 1: A sample use of the average proximity method to serve multiple viewers

$$
C V R=\frac{D S}{\sqrt{\left(\left(\frac{N H R}{N V R}\right)^{2}+1\right)} \times V D \times \tan \left(\frac{1}{60} \times \frac{\pi}{180}\right)}
$$

Similar to the use of screen size in current responsive design methods, designers can use the calculated CVR as a parameter for defining a set of media queries where each media query will correspond to a distance range that effectively defines a zone in front of the display. This means that the zones in which users are standing as well as their actual distances from the display can be used to adapt content to multiple viewers.

\section{User Study}

We conducted a user study in a controlled lab setting to evaluate the proposed adaptation model with groups of people. The goal of our experiment was two-fold. First, we wanted to examine whether the proposed model improves the user engagement, usability and viewer perception in the case of multiple viewers. Second, we aimed to evaluate how the model would compare in a multi-viewer setting with current methods used to adapt content based on display characteristics. Note that a key difference in these two methods is that the former performs a dynamic adaptation as viewers move, while the latter is a fixed adaptation. Figure 1 illustrates the sample use of the average proximity method to adapt the content to groups of viewers. For our initial study, we decided to use the average proximity method since it seems the fairest in terms of giving the same consideration to all viewers.

\subsection{Participants}

We recruited 24 participants (8 females; 20-41 (median: 23) years) for the user study. The participants were arranged in groups of three ( 8 groups). All of the participants had normal or corrected-to-normal eyesight. 


\subsection{Method and Procedure}

For each group of participants, we introduced the system and the goal of the study. We also asked their consent to record the experiment using a video camera. The experiment task was then summarised.

The task was to quickly find a specific character in a wimmelbook [7] picture, choosing characters and pictures that are well known to many people. We carefully adjusted and fitted the characters to be found so that they integrated well with other characters in the picture. Our design decision on the size of the picture for the static UI was to provide a fairly good view for both the furthest and closest viewers. Therefore, we adjusted the size of the picture to $50 \%$ of the entire screen size characteristic. For the adaptive UI, we designed the UI to resize the presentation of the picture for four zones, so that a user standing in each zone had a close to optimal view of the picture.

Since our goal was to compare the adaptive and static approaches, we decided to keep the type of adaptation as simple as possible so that the focus of the study was on the approaches and not the adaptation strategy itself.

Each group performed the tasks using the two different interfaces. Each participant in the group was equipped with a stopwatch. We showed a photo of a character and, after counting from one to three, the participants started their stopwatches and could walk around freely, while searching for the character in the picture. Once a user had found the character, they had to press the stop button and stop moving until everyone had found the character. This allowed us to identify and record the zones in which they found the character as well as how long it took each of them to find it. This procedure was repeated five times for each approach using different pictures (total: 10 conditions). The design of the studies was counterbalanced in such a way that any group was equally likely to start with one or other UI. Furthermore, the content orders in each case were randomised.

When participants had finished the tasks, they were allowed to freely move around and test the two approaches. Then, each participant filled out a questionnaire and answered some semi-structured questions about their experiences. The first part of the questionnaire consisted of questions regarding demographic information and the visual acuity of the participant. This was followed by Usability Scale (SUS) questions [8] and then a series of questions assessing user engagement. At the end, the participants also provided an overall rating on a 10-point Likert-scale for each approach.

10 questions, each on a 5 point Likert scale, were included in the SUS, allowing us to calculate a single measure of usability within the 0-100 range. We considered the SUS score as the main factor for evaluating the usability of both approaches.

To evaluate the user engagement, we used O'Brien and Toms' [9] user engagement scale (UES) which integrates a variety of user engagement factors and includes six different dimensions including Aesthetic Appeal, Endurability, Felt Involvement, Focused Attention, Novelty, and Perceived Usability. Endurability evaluates the overall success of the system, willingness of recommendation, and whether the viewer would use the system again. Aesthetic Appeal measures the visual quality of the system. Felt Involvement describes how involved the user felt with the system. Focused Attention describes to what degree the system attains the user's full attention. Novelty measures the viewer's curiosity and degree of interest. Perceived Usability reflects the user's satisfaction with the system.

By considering different dimensions, we could check from different perspectives, which approach enhances the user engagement more [9]. To evaluate the six-dimensions 
of the UES, we had eleven questions that were also used to evaluate the model with single viewers [1]. The questions were on a 5-point Likert-scale and we evaluated each dimension by summing the received scores of the corresponding questions.

To compare the attributes of the static and adaptive approaches, we used relatedsamples Wilcoxon signed rank tests. Moreover, when the assumptions were met (i.e. no normality violation as tested by Shapiro-Wilk test ( $p>0.05)$, etc. ), we used repeated measure ANOVA. We considered $\mathrm{p}=0.05$ as the minimum significance level.

We conducted our experiments using a 27" LED display, configured in landscape mode (see Fig. 2). The ResponDis framework was configured based on the display information, i.e. $\mathrm{DS}=27, \mathrm{NHR}=1920$, and $\mathrm{NVR}=1080$. For our experiment, we used the default setting "averageProximity" to adapt the UI. We also used one Kinect which can simultaneously track a maximum of six people.

\subsection{Results}

\subsubsection{Viewer Perception}

The average time measurements of the repeated experiments for five different pictures, showed a trend $(Z=1.914, p=0.056)$ toward less time required to find the character using the adaptive approach (Median $=4.5870 s$ ) compared to the static approach $($ Median $=6.1390 s)$. This result suggests that the adaptive method improved the participants' perception of the content by $25.28 \%$.

Moreover, the ending zones where the participants ended up finding the character for both static (Median =2.2) and adaptive (Median =3) approaches, were statistically significantly different $(\mathrm{Z}=-3.617, \mathrm{p}<0.001)$. Therefore, using the adaptive approach par-

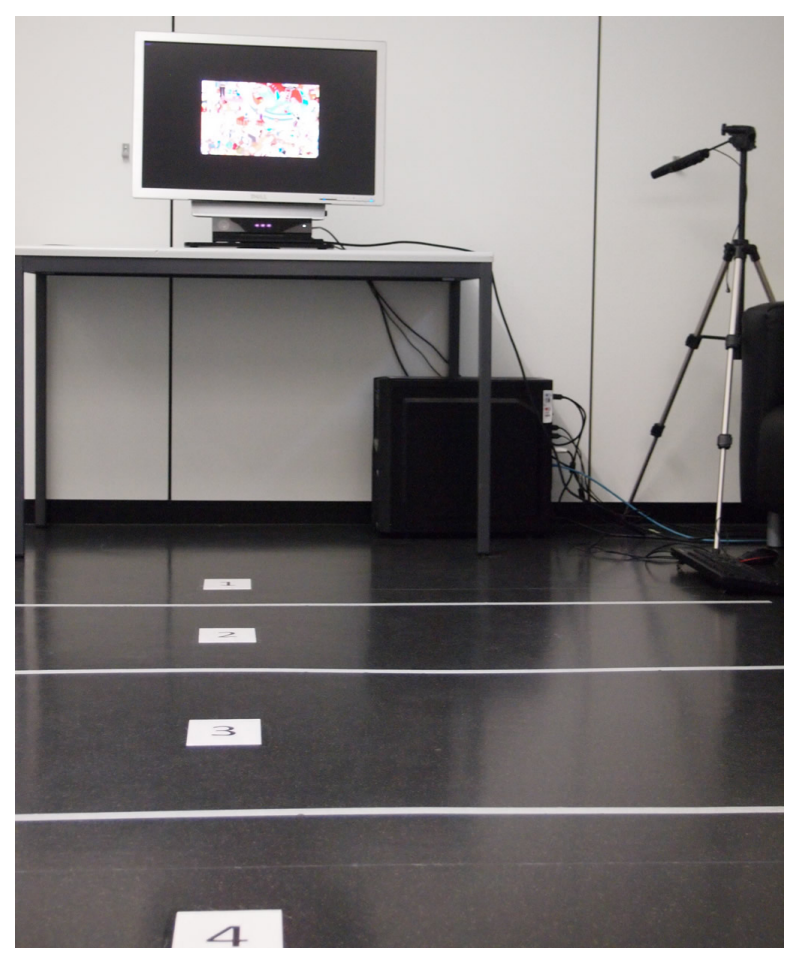

Figure 2: The study setup (picture published earlier in Tafreshi et al. [1]). 


\begin{tabular}{|l|l|l|l|c|}
\hline $\begin{array}{l}\text { Factors of Engage- } \\
\text { ment }\end{array}$ & Median & Z-value & p-Value & Conclusion \\
\hline Aestethic Appeal & Adaptive: 4, Static: 3 & $\mathrm{Z}=-1.624$ & $\mathrm{p}=0.104$ & $\mathbf{n . s}$ \\
\hline Endurability & Adaptive: 7, Static: 6 & $\mathrm{Z}=-1.844$ & $\mathrm{p}=0.065$ & $\mathbf{n . s}$ \\
\hline Felt Involvement & Adaptive: 8, Static: 7 & $\mathrm{Z}=-2.777$ & $\mathrm{p}=0.005$ & $\checkmark$ \\
\hline Focused Attention & Adaptive: 7, Static: 8 & $\mathrm{Z}=0.253$ & $\mathrm{p}=0.800$ & $\mathbf{n . s}$ \\
\hline Novelty & $\begin{array}{l}\text { Adaptive: 8, Static: } \\
3.5\end{array}$ & $\mathrm{Z}=-3.777$ & $\mathrm{p}<0.001$ & $\checkmark$ \\
\hline Perceived Usability & $\begin{array}{l}\text { Adaptive: 4.5, Static: } \\
4\end{array}$ & $\mathrm{Z}=-0.986$ & $\mathrm{p}=0.324$ & $\mathbf{n . s}$ \\
\hline
\end{tabular}

Table 1: Comparison of different dimensions of user engagement for adaptive and static approaches. $\checkmark$ : the adaptive approach performs better; n.s.: no difference was found.

ticipants needed to move less to adapt themselves to the picture on the display compared to the static approach.

\subsubsection{User Engagement}

The results of the statistical analysis to evaluate the difference between the approaches on different dimensions of the user engagement are presented in Table 1. The comparison column indicates which approach had a statistically significantly higher value, or whether there was no statistically significant difference. The sign $\checkmark$ mark in the conclusion column shows significant findings with $\mathrm{p} \leq 0.05$ if the adaptive approach performed better than the static model.n.s. marks non-significant differences. There was no case where the static approach was superior.

\subsubsection{Usability and Overall rating}

There was no statistically significant difference between the SUS score of the adaptive $($ Median $=75)$ and static $($ Median $=77.5)$ approaches, $Z=1.333, p=0.182$. Further, the overall rating on a 10 -point Likert scale showed no statistically significant difference between the two approaches, $Z=-0.185, p=0.853$. However, the adaptive approach achieved a relatively higher score $($ Median $=7)$ compared to the static approach $($ Median $=6)$.

\subsubsection{User Feedback}

Participants considered the static UI as the "state of the art, nothing new". (P 1.3). Another participant noted: "It was just a static picture. There is nothing special about that." (P 3.3). They also highlighted one of the issues of static UI design: "This is how we know it from everywhere. I just have to go very close to see the small things on it." (P 2.1). "The pictures were just small, I do not see the point. ...” (P 3.2). 
Using the adaptive design, some "... didnt understand what it adapts to" (P 6.1). One participant wrote: "At first, I did not understand that the adaptive display was changing the screen size in line with the distance. I was far from the screen. I thought it was randomly re-sizing to make the task more difficult. This was probably because we were multiple users, and the screen was trying to adapt to all of us at the same time. In the end I found it confusing why it was re-sizing." (P 8.3). Many would have preferred a smoother transition between the zones: "The state changes are too coarse. ..." (P 3.2) and many suggested: "some kind of smoothed transition and/or a way to prevent too fast switching between two states should be implemented for a calmer user experience”. (P 2.3).

Our adaptive approach was completely new to users and therefore a few of them were confused, irritated and looking for a familiar feature: “it was frustrating to see the image get smaller" ( $\mathrm{p}$ 8.1). The abrupt transition “... distracts from whatever search pattern I was on. I had to recollect myself for a moment and find out where I was again.” (P 8.2). However, other participants found that "... the adaptive display is very helpful for people who do not see very well." (P 2.1). In addition, they found that the images "... were easier to see because of the state changes ...” (P 3.2). Some future work was also suggested: “... it would be interesting to see the difference it makes while trying to read something." (P 2.3). Another participant noted that: "I think the time to find the character also depends on the user's familiarity with the character. I.e. because I am familiar with Pink Panther, it was easy for me to spot it." (P 6.2). Overall the adaptive approach was preferred: "... I would really prefer the adaptive approach. It would help for example at the tram station." (P 2.1).

Choosing an appropriate method for groups of viewers was found challenging and requires further investigation as one participant mentioned: "I feel that the adaptive display would be better suited for an individual, not a group.". (P 5.1). One participant also reported one of the difficulties in decision making for serving multiple viewers: "The only drawback of the adaptive approach in my opinion is the flickering due to multiple people interacting and the fact that 2 people, one standing far away, the second one close result in a state where one of those cannot see what he should." (7.3).

\section{Discussion}

We observed a trend toward requiring less time to find the character using the adaptive approach. While the insignificant improvement could be due to the approach, it might also be a result of the observed behaviour of the participants: Some participants were eager to find the character as fast as possible, and members of a group started competing against each other. In contrast, other groups walked around slowly, trying to not disturb one another.

The analysis of the zones where the participants ended up finding the character proved that, using the adaptive approach, participants did not have to walk as close to the display. This can be attributed to the fact that the display adapted itself to the viewers, rather than the participants having to adapt their position to the display. Therefore, the adaptive approach could be more effective in a public setting, as it requires less effort from the viewers.

The issue of engaging the viewers of pervasive display systems is a well-known problem [10-12]. We observed a systematic difference in favour of the adaptive approach on 
the Felt Involvement and Novelty factors of user engagement. The higher level of felt involvement is likely due to the adaptation of the UI according to the position of the viewer. The difference in the novelty can of course be accredited to the fact that current UI designs use the static approach and participants had not previously encountered dynamic, proximity-based adaptation.

Furthermore, viewers perceived using the adaptive approach to be more worthwhile. However, it was observed that the adaptive approach could also be more confusing. Therefore, the "average distance" strategy is probably not the best and other methods need to be explored for serving multiple viewers. Reviewing the feedback and recorded videos, the main confusion seemed to be due to the display UI flickering when a participant moved forward and backward asymmetrically. Viewers also did not expect the abrupt change of the UI. Some participants suggested that there should be a smoother transition between the states. Also, it was clear that some participants did not realise how the UI actually adapted to multiple viewers. Nonetheless, we found no systematic difference between the approaches about the feeling of being annoyed.

The usability scores of both approaches were above average but not statistically significantly different from each other. Therefore, using either of the approaches, there is no risk of a low usability effect leading to low user engagement.

Although, the adaptive approach achieved a higher overall rating and provided viewers with a better view, we observed no statistically significant difference between the overall rating of the approaches.

\section{Conclusion and Future Work}

Unlike modern personal devices that have touch input [13], some larger displays are out of reach or support no direct input on the display surface. We have presented a user study that considers the average proximity of viewers as an input to handle proximitybased adaptation of content to multiple viewers of public displays. In our study, we used the average proximity of viewers as an input to a previously proposed proximity-based adaptation model that integrates viewer distance proximity as an additional dimension for responsive web design. Although the results of our study with multiple viewers showed an enhancement in favour of the adaptive method, the improvement achieved was not as significant as that found in the previous single-viewer user study [1]. Therefore, we plan to investigate alternative methods and refinements for proximity-based adaptation in multiple viewer settings in future work.

\section{References}

[1] Amir Esmaeil Sarabadani Tafreshi, Kim Marbach, and Moira C. Norrie. ProximityBased Adaptation of Web Content on Public Displays. In International Conference on Web Engineering (ICWE), pages 282-301. Springer International Publishing, 2017. DOI: 10.1007/978-3-319-60131-1 6 .

[2] Ben Frain. Responsive Web Design with HTML5 and CSS3. Packt Publishing Ltd, 2015. 
[3] Amir E. Sarabadani Tafreshi, Adrian Wicki, and Gerhard Tröster. RDSpeed: Development Framework for Speed-Based Adaptation of Web Content on Public Displays. In 26th International Conference in Central Europe on Computer Graphics, Visualization and Computer Vision (WSCG). WSCG, 2018.

[4] Till Ballendat, Nicolai Marquardt, and Saul Greenberg. Proxemic Interaction: Designing for a Proximity and Orientation-aware Environment. In ACM International Conference on Interactive Tabletops and Surfaces (ITS), pages 121-130. ACM, 2010. DOI: 10.1145/1936652.1936676.

[5] Jakub Dostal, Uta Hinrichs, Per Ola Kristensson, and Aaron Quigley. SpiderEyes: Designing Attention- and Proximity-aware Collaborative Interfaces for Wall-sized Displays. In Proceedings of the 19th International Conference on Intelligent User Interfaces, IUI '14, pages 143-152, 2014. DOI: 10.1145/2557500.2557541.

[6] Miaosen Wang, Sebastian Boring, and Saul Greenberg. Proxemic peddler: A public advertising display that captures and preserves the attention of a passerby. In Proceedings of the 2012 International Symposium on Pervasive Displays (PerDis), pages 3:1-3:6. ACM, 2012. DOI: 10.1145/2307798.2307801.

[7] Cornelia Rémi. Reading as playing. Emergent Literacy: Children's books from 0 to 3, 13, 2011.

[8] Jeff Sauro. A Practical Guide to the System Usability Scale: Background, Benchmarks \& Best Practices. Measuring Usability LLC, 2011.

[9] Heather L OBrien and Elaine G Toms. Examining the Generalizability of the User Engagement Scale (UES) in Exploratory Search. Information Processing \& Management, 49(5):1092-1107, 2013.

[10] Amir E. Sarabadani Tafreshi and Moira C. Norrie. Screenpress: A powerful and flexible platform for networked pervasive display systems. In Proceedings of the 6th ACM International Symposium on Pervasive Displays (PerDis), pages 13:1-13:8. ACM, 2017. DOI: 10.1145/3078810.3078813.

[11] Amir E. Sarabadani Tafreshi, Milan Bombsch, and Gerhard Tröster. Chained Displays: Configuration of Multiple Co-Located Public Display. International Journal of Computer Networks \& Communications (IJCNC), 10(3), 2018. DOI: $10.5121 / \mathrm{ijcnc} .2018 .10301$.

[12] Amir E. Sarabadani Tafreshi, Andrea Soro, and Gerhard Tröster. Automatic, Gestural, Voice, Positional, or Cross-Device Interaction? Comparing Interaction Methods to Indicate Topics of Interest to Public Displays. In Frontiers in ICT. Frontiers, 2018.

[13] Amir E. Sarabadani Tafreshi, Sara C. Sarabadani Tafreshi, and Amirehsan Sarabadani Tafreshi. Tiltpass: Using device tilts as an authentication method. In Proceedings of the 2017 ACM International Conference on Interactive Surfaces and Spaces (ISS), pages 378-383. ACM, 2017. DOI: $10.1145 / 3132272.3134112$. 